\title{
Beneficial effect of pramipexole for motor function and depression in Parkinson's disease
}

\author{
Osamu Kano',2 \\ Ken Ikeda ${ }^{2}$ \\ Tetsuhito Kiyozuka² \\ Konosuke Iwamoto ${ }^{2}$ \\ Hirono Ito ${ }^{2}$ \\ Yuji Kawase 2 \\ Ryuta Sato ${ }^{2}$ \\ Toshiki Fujioka² \\ Yo Araki \\ Shigeji Baba ${ }^{2}$ \\ Yasuo Iwasaki ${ }^{2}$ \\ 'Department of Neurology, Methodist \\ Neurological Institute, Houston, TX, \\ USA; ${ }^{2}$ Department of Neurology, Toho \\ University Omori Medical Center, \\ Tokyo, Japan
}

\begin{abstract}
We examined whether pramipexole (PPX) can influence depressive scale in normal and mild depressive parkinsonian patients. In an open study of PPX as an add-on to L-dopa therapy or single administration, 36 nondemented outpatients with Parkinson's disease (PD) were entered first. All were in the stage II or III of Hoehn and Yahr scale (H\&Y). PPX were started at $0.125 \mathrm{mg} /$ day and daily doses were increased to $1.5 \mathrm{mg} / \mathrm{day}$. At 3 months after PPX treatment, patients were re-evaluated. Hamilton Depression Rating Scale (HAM-D), Unified Parkinson's Disease Rating Scale III, H\&Y stage, and freezing of gait questionnaire were compared in patients before and after PPX treatment. These scores were significantly improved after PPX administration. There were no correlations between HAM-D and those motor functions. We suggest that PPX treatment has antidepressant effects in depressive PD patients and also ameliorates HAM-D score in nondepressive PD patients in addition to motor function.
\end{abstract}

Keywords: Parkinson disease, pramipexole, motor function, depression, antidepressant effect

\section{Introduction}

Depression occurs in about $40 \%-45 \%$ of patients with Parkinson's disease (PD) and reduces both the subjective and objective quality of life independent of motor deficits (Cummings et al 1999; Kupio et al 2000). Depressive symptoms precede those of motor function in 12\%-37\% of patients with PD (Taylor et al 1986). The pathological feature of $\mathrm{PD}$ reveals degeneration of dopaminergic neurons in the striato-nigral system. In addition, the limbic system is also involved in PD (Lemke et al 2004). Pramipexole (PPX), $\mathrm{D}_{2} / \mathrm{D}_{3}$ receptor agonist acts on $\mathrm{D}_{3}$ versus $\mathrm{D}_{2}$ receptors in the regions of the brain that play a key role in the etiology of depression (Mayberg et al 1995). These mechanisms could explain the effectiveness of PPX on depression in animal experiments (Willner et al 1994; Maj et al 1997) and in patients with major depressive disorder (Szegedi et al 1997; Corrigan et al 2000).

Recent European studies show that PPX treatment is beneficial for patients with depression and PD and PPX is more effective on depression in PD as compared with sertraline, an antidepressant (Rektorova et al 2003; Barone et al 2006). Furthermore, patients with refractory bipolar depression were treated with PPX as add-on therapy to various mood stabilizers (Goldberg et al 2004; Zarate et al 2004; Aiken 2007).

Thus, the antidepressant effect of PPX in patients with a major depressive disorder has already been studied and PPX seems to be more powerful compared with other ergoline preparations such as bromocriptine, pergolide, and cabelgoline (Boyd 1995; Reeves et al 1997; Baeza et al 2002; Rektorova et al 2003). In this paper we studied how PPX treatment influences depressions and motor scales in PD patients. 


\section{Materials and methods}

All patients underwent neurological evaluation. We included patients with idiopathic PD according to the UK Parkinson's Disease Society Brain Bank Clinical Diagnostic Criteria (Daniel et al 1993), and obtained informed consent. The duration of the trial was from March 1st to July 31st 2006. In this prospective, observational, open study, patients with moderate and severe depressive episode defined according to the 10th revision of the International Classification of Diseases were excluded for administration of PPX. In an open study of PPX as an add-on to L-dopa therapy or single administration, PPX was started at $0.125 \mathrm{mg} /$ day and daily doses were increased to $1.5 \mathrm{mg}$ /day. Patients on L-dopa therapy continued to take the same dosage of levodopa which was kept fixed during the study. At 3 months after PPX treatment, patients were re-evaluated. Hamilton Depression Rating Scale (HAM-D) (Hamilton 1960), Unified Parkinson's Disease Rating Scale (UPDRS) III (Fahn et al 1987), Hoehn and Yahr (H\&Y) stage (Hoehn et al 1967), and freezing of gait questionnaire (FOGQ) (Giladi et al 2000) were compared in patients before and after PPX treatment. Statistical analysis used Wilcoxon signed rank test, Kruskal Wallis test, with SPSS for Windows version 13.0 (SPSS Inc., Chicago, IL, USA).

\section{Results}

Initially 36 PD outpatients without dementia were entered. A total of 31 patients (16 women and 15 men) were eligible for this trial. The clinical background of 31 patients (16 women and 15 men) is shown in Table 1. Drop-outs due to adverse events occurred in 3 patients (nausea, aggravation of dyskinesia), but all were not serious, and 2 patients moved to another town. The mean age (SD) was 71.2 (8.3) years (70.9 [8.6] in women and 71.4 [8.2] in men). The mean disease duration (SD) was 4.4 (7.3) years (4.0 [3.9] in women and 4.7 [3.6] in men). All were able to walk independently (H\&Y stage II or III). Twenty-nine patients received L-dopa. Two patients had PPX alone. None were treated with any antidepressants. Changes of neurological evaluation were shown in Table 2. After PPX administration, HAM-D score was declined. Motor function scores were also significantly improved. There were no statistical correlations among HAM-D, UPDRS III, H\&Y stage, and FOGQ.

\section{Discussion}

We excluded patients with moderate and severe depressive episode. PPX doses used were lower than $1.5 \mathrm{mg} / \mathrm{day}$.
Table I Clinical background of patients

\begin{tabular}{llll}
\hline & Total & Women & Men \\
\hline Age (years) & $71.2(8.3)$ & $70.9(8.6)$ & $71.4(8.2)$ \\
Duration of disease (years) & $4.4(7.3)$ & $4.0(3.9)$ & $4.7(3.6)$ \\
H\&Y stage & $2.5(0.6)$ & $2.5(0.7)$ & $2.5(0.5)$ \\
L-dopa doses (mg/day) & $217.7(91.8)$ & $190.6(100.4)$ & $246.7(74.3)$ \\
Pramipexole doses (mg/day) & $1.1(0.5)$ & $1.1(0.5)$ & $1.1(0.5)$ \\
\hline
\end{tabular}

Notes: Data are shown as mean (SD).

Those relative low doses of PPX improved HAM-D score significantly. In relationship between other dopamine agonists and mental changes, bromocriptine worsened psychotic symptoms in patients suffering from schizophrenia, other psychotic disorders, or psychotic depression (Pearson 1981). Cabergoline can be used similarly to bromocriptine in PD, and only a few reports discussed the efficacy of use in psychotic symptoms (Baeza et al 2002). Pergolide is well used in PD, but this dopamine agonist is reported to have no efficacy on depression in PD patients by national multicenter prospective randomized studies (Rektorova et al 2003). In contrast, ropinirole, which is a nonergot dopamine agonist, as well as PPX showed an antidepressant effect, but no reports showed a comparison of antidepressant efficiency between ropinirole and PPX (Pahwa et al 2007).

PPX had significantly improved not only depressive parkinsonian patients but also bipolar depression (Corrigan et al 2000; Goldberg et al 2004; Zarate et al 2004). PPX was well tolerated and was significantly more effective in reducing severity of depression and increasing numbers of responders (Goldberg et al 2004; Zarate et al 2004). These effects may relate to PPX's preference for $\mathrm{D}_{3}$ versus $\mathrm{D}_{2}$ receptors and neurotrophic properties. Furthermore, $\mathrm{D}_{3}$ receptors may have an anatomic distribution which plays an important role in neuronal circuits implicated in depressive states.

According to a detailed survey of antidepressant drugs in PD by the Parkinson Study Group in the USA (Richard et al 2000), the administration of selective serotonin reuptake inhibitors (SSRI) usually serves as the first-choice treatment.

Corrigan and colleagues (2000) reported that PPX for patients with major depression at the $1.0 \mathrm{mg}$ per day dose had significantly improvement over baseline compared to the placebo measure of the HAM-D, MontgomeryAsberg Depression Rating Scale, and Clinician's Global Impressions - Severity of Illness scale, and the most obvious 
Table 2 Results of depression scale and motor function

\begin{tabular}{lll}
\hline & Baseline & Endpoint \\
\hline HAM-D & $11.7(6.1)$ & $7.5(5.4)^{*}$ \\
H\&Y stage & $2.5(0.6)$ & $2.1(1.1)^{*}$ \\
UPDRS III & $26.8(6.2)$ & $20.6(7.0)^{*}$ \\
Freezing index A & $12.1(11.1)$ & $10.0(9.2)^{*}$ \\
Freezing index B & $5.4(5.3)$ & $4.2(4.1)^{*}$ \\
\hline
\end{tabular}

Notes: Data are shown as mean (SD); $* \mathrm{P}<0.0 \mathrm{l}$ by Wilcoxon signed rank test between baseline and endpoint. There were no statistical significances of HAM-D score and other motor scales.

improvement was seen in the PPX $5.0 \mathrm{mg}$ group, although a substantial dropout rate increased (Corrigan et al 2000). A recent European study reported that PPX is more effective on depression in PD as compared with the antidepressant sertraline (Barone et al 2006).

In the present study, the HAM-D score was reduced during treatment with PPX and those antidepressant effects did not depend on motor function scale. Previous European experience (Lemke et al 2006) and our studies suggest that PPX has benefits for depressive symptoms in PD patients. These results support that the original serotonergic and noradrenergic hypotheses do not fully account for the neurobiology of depression or mechanism of action of effective antidepressants. Roy and colleagues (1985) found lower cerebrospinal fluid homovanillic acid (HVA) which was known as dopamine metabolite in depressed subjects than in normal subjects. Also, direct measurement of brain monoamine metabolites from the internal jugular vein of treatment-resistant depressed patients revealed low HVA levels that were highly correlated with illness severity (Lambert et al 2000).

Thus, these results support the monoaminergic theories of depression, which hold that dysregulation of systems involving dopamine, in addition to serotonin and norepinephrine, may be involved in major depression. The mechanism of action of currently available antidepressants is to re-equilibrate one or more neurotransmitter systems and restore relative efficiency at one or more synaptic sites, such as by altering postsynaptic receptor sensitivity (Siever et al 1985; Willner et al 2005). It is believed that PPX acts as a direct agonist at the postsynaptic receptor, thereby relieving some symptoms of depression.

Since the HAM-D score and motor function were improved after administration of PPX, and there was no correlation between HAM-D and motor functions, this indicates improvement of HAM-D score was not due to an improvement in motor functions.
We conclude that PPX is a useful strategy in improving of depressive state in PD. Further long-term study of PPX is needed to determine whether this dopamine agonist can prevent upcoming depression in PD.

\section{References}

Aiken CB. 2007. Pramipexole in psychiatry: a systematic review of the literature. J Clin Psychiatry, 68:1230-6.

Baeza PI, Goikolea AJ, Parellada RE. 2002. Is cabergoline a better drug to inhibit lactation in patients with psychotic symptoms? J Psychiatry Neurosci, 27:54.

Barone P, Scarzella L, Marconi R, et al; Depression/Parkinson Italian Study Group. 2006. Pramipexole versus sertraline in the treatment of depression in Parkinson's disease: A national multicenter parallel-group randomized study. J Neurol, 253:601-7.

Boyd A. 1995. Bromocriptine and psychosis: a literature review. Psychiatr $Q, 66: 87-95$.

Corrigan MH, Denahan AQ, Wright CE, et al. 2000. Comparison of pramipexole, fluoxetine, and placebo in patients with major depression. Depress Anxiety, 11:58-65.

Cummings JL, Masterman DL. 1999. Depression in patients with Parkinson's disease. Int J Geriatr Psychiatry, 14:711-18.

Daniel SE, Lees AJ. 1993. Parkinson's Disease Society Brain Bank. London: overview and research. J Neurol Transm, 39:165-72.

Fahn S, Marsden CD, Calne DB, et al. (eds). 1987. Recent developments in Parkinson's disease. Vol 2. Florham Park, NJ: Macmillan Health Care Information, pp 153-63.

Giladi N, Shabtai H, Simon ES, et al. 2000. Construction of freezing of gait questionnaire for patients with Parkinsonism. Parkinsonism Relat Disord, 6:165-70.

Goldberg JF, Burdick KE, Endick CJ. 2004. Preliminary randomized, double-blind, placebo-controlled trial of pramipexole added to mood stabilizers for treatment-resistant bipolar depression. Am J Psychiatry, 161:564-6.

Hamilton, M. 1960. A rating scale for depression. J Neurol Neurosurg Psychiatry, 23:56-62.

Hoehn MM, Yahr MD. 1967. Parkinsonism: onset, progression and mortality. Neurology, 17:427-42.

Kuopio AM, Marttila RJ, Helenius H, et al. 2000. The quality of life in Parkinson's disease. Mov Disord, 15:216-23.

Lambert G, Johansson M, Agren H, et al. 2000. Reduced brain norepinephrine and dopamine release in treatment-refractory depressive illness: Evidence in support of the catecholamine hypothesis of mood disorders. Arch Gen Psychiatry, 57:787-93.

Lemke MR, Fuchs G, Gemende I, et al. 2004. Depression and Parkinson's disease. J Neurol, 251:24-7.

Lemke MR, Brecht HM, Koester J, et al. 2006. Effects of the dopamine agonist pramipexole on depression, anhedonia and motor functioning in Parkinson's disease. J Neurol Sci, 248:226-70.

Maj J, Rogoz Z, Skuza G, et al. 1997. The behavioral effects of pramipexol, a novel dopamine receptor agonist. Eur J Pharmacol, 324:31-7.

Mayberg HS, Solomon DH. 1995. Depression in Parkinson's disease: a biochemical and organic viewpoint. Adv Neurol, 65:49-60.

Pahwa R, Stacy MA, Factor SA, et al. 2007. Ropinirole 24-hour prolonged release: randomized, controlled study in advanced Parkinson disease. Neurology, 68:1108-15.

Pearson KC. 1981. Mental disorders from low-dose bromocriptine. N Engl $J$ Med, 305:173.

Reeves RR, Pinkofsky HB. 1997. Postpartum psychosis induced by bromocriptine and pseudoephedrine. J Fam Pract, 45:164-6.

Rektorova I, Rektor I, Bares M, et al. 2003. Pramipexole and pergolide in the treatment of depression in Parkinson's disease: a national multicentre prospective randomized study. Eur J Neurol, 10:399-406.

Richard IH, Kurlan R. 2000. A survey of antidepressant usage in Parkinson's disease. Neurology, 2:263-73. 
Roy A, Pickar D, Linnoila M, et al. 1985. Cerebrospinal fluid monoamine and monoamine metabolite concentrations in melancholia. Psychiatry Res, 14:281-92.

Siever LJ, Davis KL. 1985. Overview: toward a dysregulation hypothesis of depression. Am J Psychiatry, 142:1017-31.

Szegedi A, Hillert A, Wetzel H, et al. 1997. Pramipexole, a dopamine agonist, in major depression: antidepressant effects and tolerability in an open-label study with multiple doses. Clin Neuropharmacol, 20:S36-45.

Taylor A, Saint-Cyr JA, Lang AE, et al. 1986. Parkinson's disease and depression: a critical re-evaluation. Brain, 109:279-92.
Webster J. 1996. A comparative review of the tolerability profiles of dopamine agonists in the treatment of hyperprolactinaemia and inhibition of lactation. Drug Safety, 14:228-38.

Willner P, Lappas S, Cheeta S, et al. 1994. Reversal of stress induced anhedonia by the dopamine receptor agonist, pramipexole. Psychopharmacology (Berl), 115:454-62.

Willner P, Hale AS, Argyropoulos S. 2005. Dopaminergic mechanism of antidepressant action in depressed patients. J Affect Disord, 86:37-45.

Zarate CA Jr, Payne JL, Singh J, et al. 2004. Pramipexole for bipolar II depression: a placebo-controlled proof of concept study. Biol Psychiatry, 56:54-60. 